\title{
PHOTONIC DEVICES WITH SILOXANE MEMBRANES
}

Expansion of polymer nanotechnologies accelerated the polymer photonics. This technology brings novel interesting results with simple and cheap solutions. Promising candidates are siloxane materials as polydimethyl siloxane (PDMS) and polydimethyldiphenyl siloxane (PDMDPS) with unique mechanical and optical properties. In this paper we present laser lithography technologies in a combination with siloxane embossing for fabrication of different grating structures with periodical and quasiperiodical symmetry. We present the concept of siloxane based thin membranes with patterned surface as an effective diffraction element for modification of radiation pattern diagram of light emitting diodes (LEDs) and enhancement of angular detection properties of photodiodes. Also the combination of PDMDPS and PDMS was used for fabrication of a waveguide structure with surface relief Bragg grating. Optical properties of fabricated LEDs are investigated by near-and far-field measurements. Transmission properties of fabricated waveguide are investigated by optical spectrum analyzer.

Keywords: Photonic crystal, photonic quasicrystal, light emitting diode, waveguide, light extraction efficiency

\section{Introduction}

Polymer based photonics bring us novel interesting results with simple and cheap solutions. Creating devices with flexible features was a challenge for polymer photonics where polymer materials are used instead of semiconductor. The siloxanes are flexible silicon based elastomers with unique properties which make it attractive for optical applications. In the waveguide optics, we can use polymer siloxanes for fabrication of the optical waveguides [1], optical fibres [2], optical sensors [3] and optical waveguides with surface relief Bragg gratings (SRBG). Major research was focused on improvement of light extraction efficiency (LEE) and radiation pattern modification of light emitting diodes (LED). Many groups used implementation of photonic crystal $(\mathrm{PhC})$ in the LED surface to change these optical properties [4, 5]. Generally, the current technologies try to pattern the surface of the LED by combination of lithography and surface etching or with colloidal crystals [6]. Another interesting way is using the thin siloxane membranes with the PhC structures which can be applied directly on the LED surface [7]. This technique offers a new solution how to easily modify the LED properties as LEE and radiation pattern diagram.

Good candidates are siloxane materials such as polydimethyl siloxane (PDMS) and polydimethyldiphenyl siloxane (PDMDPS) with unique mechanical and optical properties. These polymers are elastic (elongation $120 \%$ ), transparent for visible and near infrared light, thermal stabile and biocompatible [8, 9]. In this paper, we present the results achieved by siloxane based technologies in a combination with laser lithography and siloxane embossing with resolution achieving hundreds of nanometers. Laser interference lithography in Mach-Zehnder configuration is able to create periodical or quasiperiodical $\mathrm{PhC}(\mathrm{PQC})$ structures in two dimensions. In the combination with embossing technique, we achieved novel polymer $\mathrm{PhC}$ structures. We proposed the concept of thin polymer membranes with patterned $\mathrm{PhC}$ structure for improving LEE and modification of radiation pattern diagram of LEDs. Also the combination of polymers PDMDPS and PDMS was used for fabrication of complicated planar waveguides with SR-BG, which can be used as a tunable filter or sensor.

\section{Fabrication process of siloxane membranes}

There are many techniques favorable for the planar $\mathrm{PhC}$ fabrication such as electron and ion beam lithography and nanoimprint. The fabrication technologies based on optical principles as interference lithography, direct laser writing (DLW) laser lithography and near-field scanning optical microscope

\footnotetext{
* ${ }^{1}$ Matej Goraus, ' ${ }^{1}$ ubos Suslik, 'Dusan Pudis, ' Jana Durisova, ' ${ }^{1}$ Daniel Jandura, ${ }^{2}$ Jaroslav Kovac, ${ }^{3}$ Marek Tlaczala

${ }^{3}$ Wojciech Dawidowski, ${ }^{3}$ Beata Sciana

${ }^{1}$ Department of Physics, Faculty of Electrical Engineering, University of Zilina, Slovakia

${ }^{2}$ Institute of Electronics and Photonics, Slovak University of Technology, Bratislava, Slovakia

${ }^{3}$ Faculty of Microsystem Electronics and Photonics, Wrocław University of Science and Technology, Wroclaw, Poland

E-mail: matej.goraus@fel.uniza.sk
} 
lithography are very useful for $\mathrm{PhC}$ structure preparation in two dimensions (2D) in photoresist and different semiconductor surfaces. We demonstrate basic principles of the laser interference lithography as effective tools for $\mathrm{PhC}$ structure fabrication in photoresist materials and in a combination with embossing technique of the liquid PDMS also for PDMS patterning. In our experiments, we used positive photoresists of $\mathrm{AZ} 5214 \mathrm{E}$ and S1828 G2.

\subsection{Laser interference lithography}

Laser interference lithography is promising technique for fabrication of $\mathrm{PhC}$ in a large scale area. 1D and 2D interference patterns can be produced by this technique. The design of $1 \mathrm{D}$ optical field pattern can be obtained by the interference process of two optical plane waves (Fig. 1a). The 2D interference pattern can be achieved by the multiple exposure process in a combination with the sample rotation between individual exposures (Fig. 1b, 1c). This multiple exposure technique can be used for fabrication of different 2D periodic structures in photoresist materials as well as in different III-V compounds. We can produce different symmetry of $\mathrm{PhC}$ and $\mathrm{PQC}$ structures depending on the angle of rotation of the sample in the exposure process. For square symmetry of $\mathrm{PhC}$ we used sample rotation at $90^{\circ}$. Also PQC structure with 12-fold symmetry was prepared by multiexposure process with the angle of rotation $0-360^{\circ}$ with $30^{\circ}$ step. The design of the structure can be proposed by the simple theoretical approach according to [10].

In our experimental arrangement we used Mach-Zehnder configuration (Fig. 2) to create interference optical field. As a source, we used Toptica Blue Mode laser emitted at $403 \mathrm{~nm}$. For fabrication of $\mathrm{PhC}$ structures we used the photoresist layer of AZ 5214E with thickness app. $2 \mu \mathrm{m}$. Photoresist layer was prepared by spin coating process on glass substrate using SPIN 150 coater with post-baking at $65^{\circ} \mathrm{C}$ for 2 minutes and at $100{ }^{\circ} \mathrm{C}$ for 3 minutes to remove the solvent. After exposure, the exposed photoresist sample was developed in AZ 400K developer for $10 \mathrm{~s}$ and rinsed in deionized water. For the PQC fabrication, we used S1828 G2 photoresist with thickness app. $3 \mu \mathrm{m}$ with post-baking at $100{ }^{\circ} \mathrm{C}$ for 2 minutes and developing in $\mathrm{AZ} 400 \mathrm{~K}$ for $10 \mathrm{~s}$.

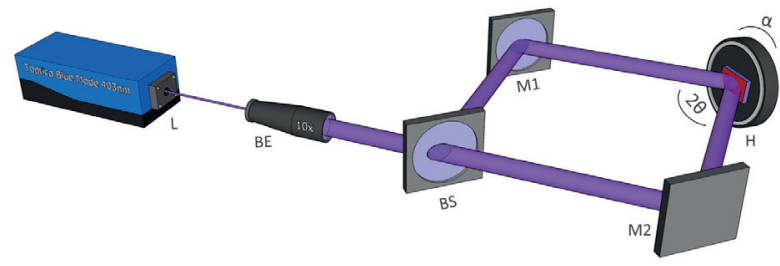

Fig. 2 Experimental arrangement of laser interference lithography in Mach-Zehnder configuration, were $L$ is the laser source, BE - beam expander, BS - 50:50 beam splitter (for wavelengths range 400-700 nm), $M 1, M 2$ - mirrors. $H$ - sample holder where $2 \theta$ is the angle between two incident beams and $\alpha$ is the angle of sample rotation

\subsection{PDMS embossing}

For the fabrication of patterned PDMS membranes, the liquid PDMS was prepared from two components of Sylgard 184 elastomer with curing agent ratio 10:1. For uniform surface, the $30 \mu \mathrm{m}$ thin PDMS layer was spin coated at $4000 \mathrm{rpm}$ on patterned photoresist samples. Subsequently, the sample was cured for 45 min at $75{ }^{\circ} \mathrm{C}$. In order to obtain PDMS membrane we used two methods. First, due to unique elastic properties of PDMS the membrane was mechanically removed from photoresist layer (Fig. 3 ). Reusability is the best advantage of this method. The other way was based on dissolving photoresist in solvent and lifting off the PDMS membrane. By using both of these ways, we obtained nearly identical PhC PDMS membranes.

\section{Fabricated photonic devices}

These technologies of fabrication of patterned siloxane membranes can be used in different optical and optoelectronical
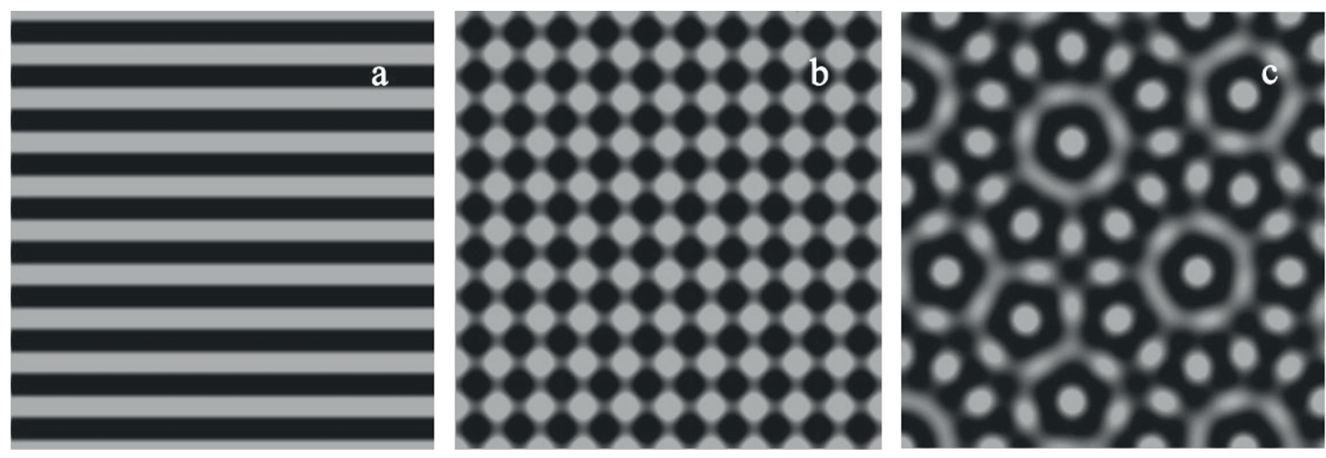

Fig. 1 Simulation of theoretical distribution of interference optical field where a) represents $1 D$ interference pattern b) $2 D$ pattern with square symmetry and c) interference pattern of PQC structure with the 12-fold symmetry 


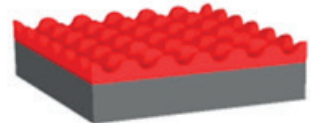

a)

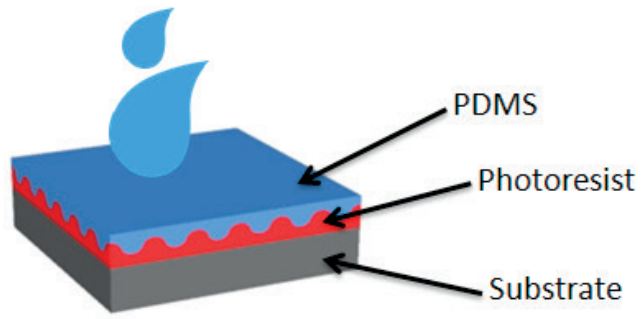

b)

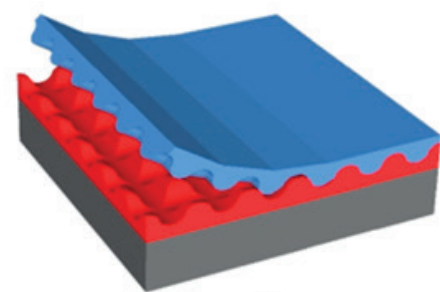

c)

Fig. 3 Embossing and removing process of embossed PDMS membranes a) substrate with photoresist master b) application of liquid PDMS and c) mechanical separation of cured membrane

applications. We focus on optical characterization of LEDs and photodiodes with patterned PDMS membranes and the PDMS waveguide with SR-BG.

\subsection{LEDs and photodiodes with PDMS membrane $\mathrm{PhC}$ and PQC}

Two-dimensional PhC of square symmetry and PQC structures were prepared by the embossing technique in the surface of thin PDMS membranes described above.

It was published, that $\mathrm{PhC}$ on the surface can lead to the light extraction efficiency improvement of LEDs and also should modify the radiation pattern diagram [11, 12]. In our experiments we patterned different PDMS membranes for direct application in LED chips. For the PDMS patterning we used the combination of photoresist patterning by interference lithography and PDMS embossing described above. After mechanical selection, the prepared PDMS membrane was directly placed on the LED chip with patterned surface oriented on the top. For the characterization we used commercial Cree LEDs with central emission at wavelength of $635 \mathrm{~nm}$. Quality of prepared embossed PDMS membranes (of thickness app. $30 \mu \mathrm{m}$ ) was analyzed by atomic force microscope (AFM) and is shown in Fig. 4. The period of the embossed structure in PDMS preserves the photoresist pattern with the period of $1600 \mathrm{~nm}$ and depth of $420 \mathrm{~nm}$. In our experiments the structures with two different symmetries were prepared to show the effect of $\mathrm{PhC}$ on radiation properties using 2D PhC structure with square symmetry (Fig. 4a) and PQC structure with 12-fold symmetry (Fig. 4b).

After the application of patterned PDMS membranes on the LED surface the radiation properties in the near field were measured by using the near-field scanning optical microscope (NSOM) with aperture-less optical fiber tips. As is shown in Fig. 5, the local surface enhancement in the near field reflects the pattern symmetry and corresponds well with AFM structure. The NSOM analysis shows the near-field of the 2D PhC structure with square symmetry and also PQC structures. Radiation properties of both structures were compared by investigation in the far field by goniophotometer measurements. Top view of the radiation pattern clearly shows the effect of higher symmetry of PQC in comparison with double symmetry of square PhC (Fig. 6a). Also an effective angular widening was documented for the PQC structure. The measured optical properties in the near and far field document the effective function of PDMS membranes on modification of radiation pattern of LEDs.

For the implementation of PDMS membranes with patterned surface PhC structure the GaAs-based photodiode with InGaAsN active region was also prepared. We used the epitaxial structure of GaAs-based photodiode grown by atmospheric pressure metal

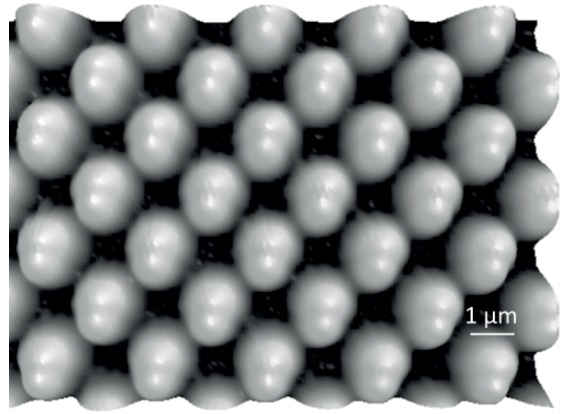

a)

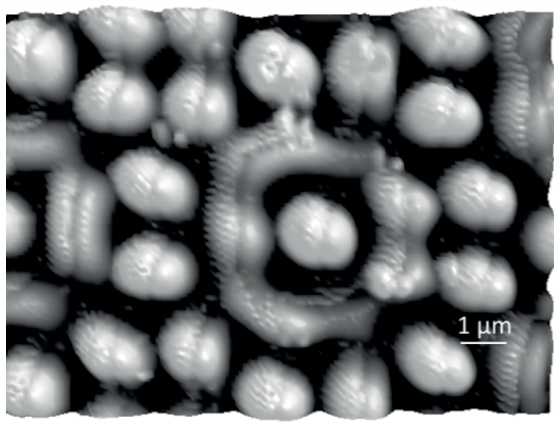

b)

Fig. 4 AFM image of a) 2D PhC structure of square symmetry and b) PQC structure with 12 fold symmetry embossed in PDMS surface 


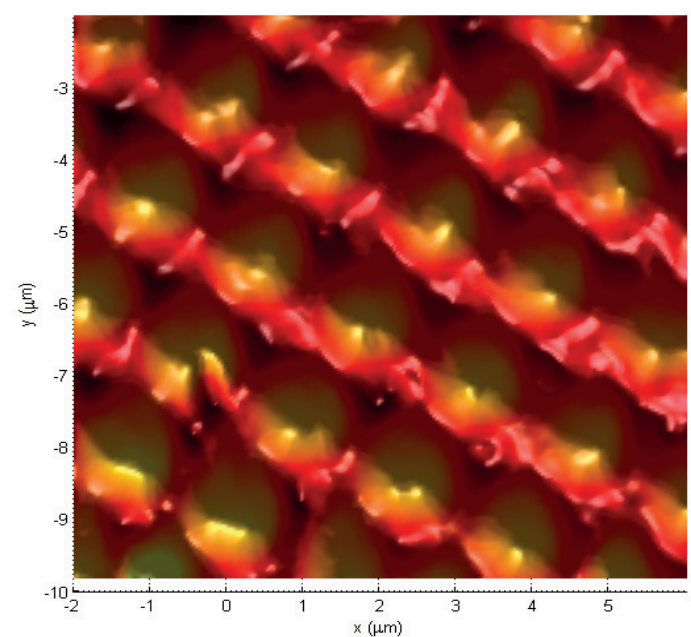

a)

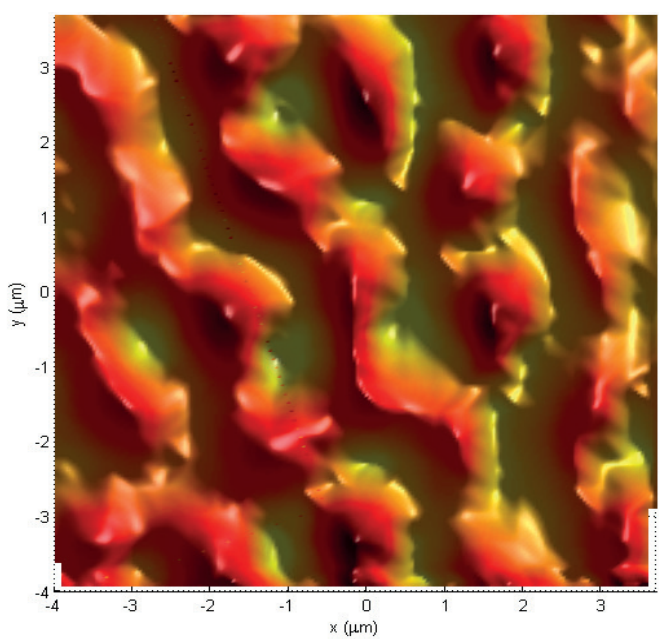

b)

Fig. 5 Near-field pattern of LED with a) PhC PDMS membrane with square symmetry and b) PQC structure with 12-fold symmetry

organic vapor phase epitaxy (AP-MOVPE). Detailed information about the growth process conditions, contact preparation and precursors were published in [13]. The active region of p-i-n GaAsbased device consist of undoped InGaAsN active layer sandwiched between silicon doped ntype GaAs buffer and $\mathrm{Zn}$ doped p-type InGaAsN layer, capped by heavily $\mathrm{Zn}$ doped p-type GaAs contact layer. On the top surface of the photodiode the patterned membrane with PhC structure of square symmetry and period of $800 \mathrm{~nm}$ was applied. The angular detection properties were measured by illumination of the photodiode with orange LED source with central emission at the wavelength of $625 \mathrm{~nm}$ (Fig. 6b). Evident square symmetry can be found in the angular photoresponse as an effect of 2D square PhC structure on the top of the photodiode. It documents the effectiveness of patterned PDMS membranes also on detection properties.

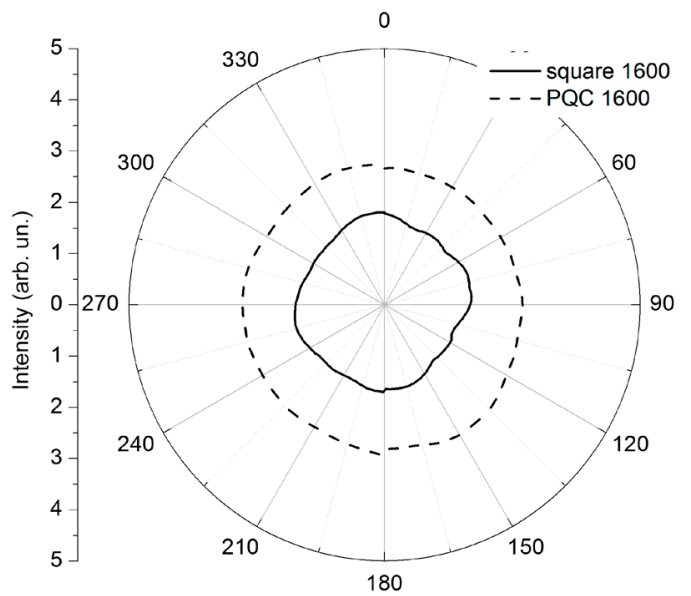

a)

\subsection{Polymer waveguide with surface relief Bragg grating}

For polymer waveguide structures with SR-BG preparation, laser interference lithography and embossing technique was used in our experiments. Patterning of thin photoresist layer was carried out by laser interference lithography in MachZehnder arrangement using Toptica Blue Mode 403 nm laser (Fig. 2).

To achieve appropriate refractive index contrast, two types of polymers were used. For the waveguide core formation we used PDMDPS and PDMS was used for the cladding formation. Onedimensional (1D) $\mathrm{PhC}$ pattern with period of $21 \mu \mathrm{m}$ was created in the first step. Next, the sample was developed in AZ400 K developer and 1D PhC pattern with period of $1.61 \mu \mathrm{m}$ was exposed perpendicularly to the first pattern in the second step. By this technological process, we created a D-shaped array of app. $10 \mu \mathrm{m}$ wide and app. $2.5 \mu \mathrm{m}$ high waveguides with SRBG

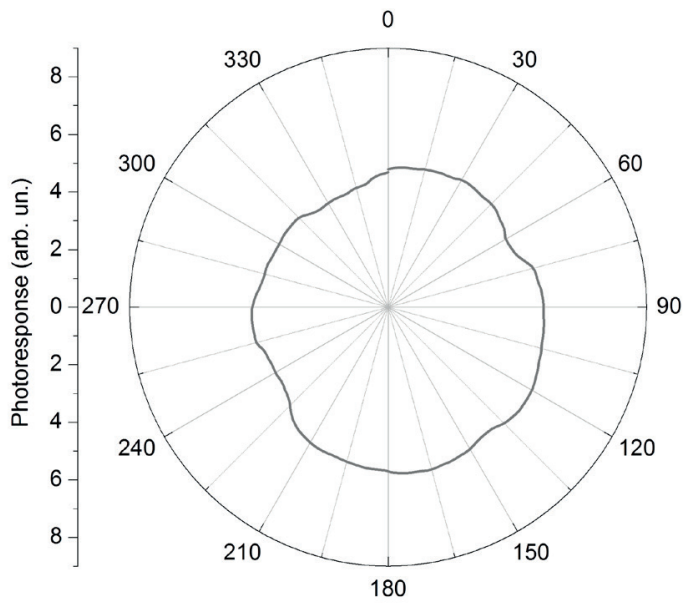

b)

Fig. 6 a) Radiation pattern of orange LED chip with applied patterned PDMS membrane with PhC and PQC structures and $b$ ) angular photoresponse measured for illumination by orange LED source 


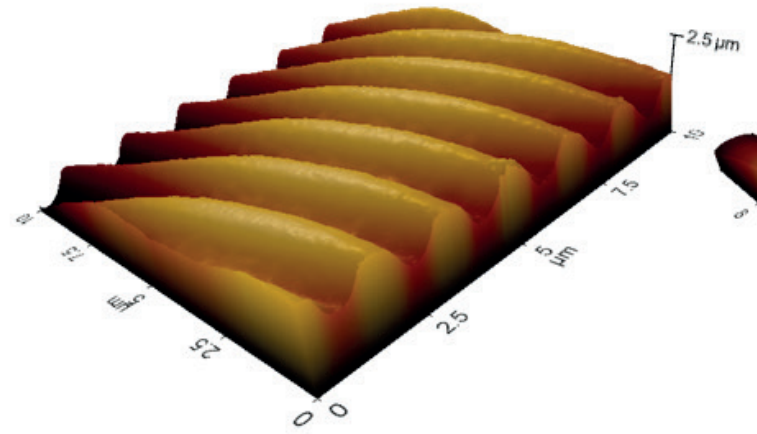

a)

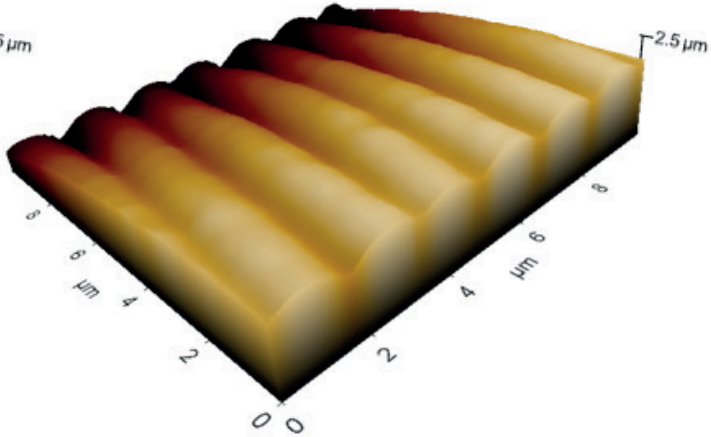

b)

Fig. 7 AFM images a) patterned photoresist master with surface Bragg grating and b) embossed PDMDPS polymer membrane with SR-BG before cladding application

in photoresist master as was documented from AFM (Fig. 7a). Array of the polymer SRBG waveguides was formed by filling the valleys with liquid PDMDPS, spin coating and heat curing. Next, the membrane was mechanically separated from the photoresist master (Fig. 7b). Cladding liquid PDMS was deposited on the patterned side of PDMDPS membrane and heat curing. By this process, we created the array of the polymer SR-BG waveguides.

The transmission properties of waveguides with SRBG were measured by light transmission characteristics using the broadband LED with central emission wavelength at $\lambda=1550 \mathrm{~nm}$. The proposed spectral dip according to equation $\lambda_{B}=2 n_{\text {eff }} \Lambda / k$ was at the wavelength $\lambda_{B}=1539 \mathrm{~nm}$, where $\lambda_{B}$ is the reflected wavelength, $n_{\text {eff }}$ is the effective refractive index of the waveguide, $\Lambda$ is the period of the grating and $k$ is the order of the reflection. The transmission spectral characteristic shows evident dip at the wavelength of $1540 \mathrm{~nm}$, what is in a good coincidence with the proposed dip (Fig. 8).

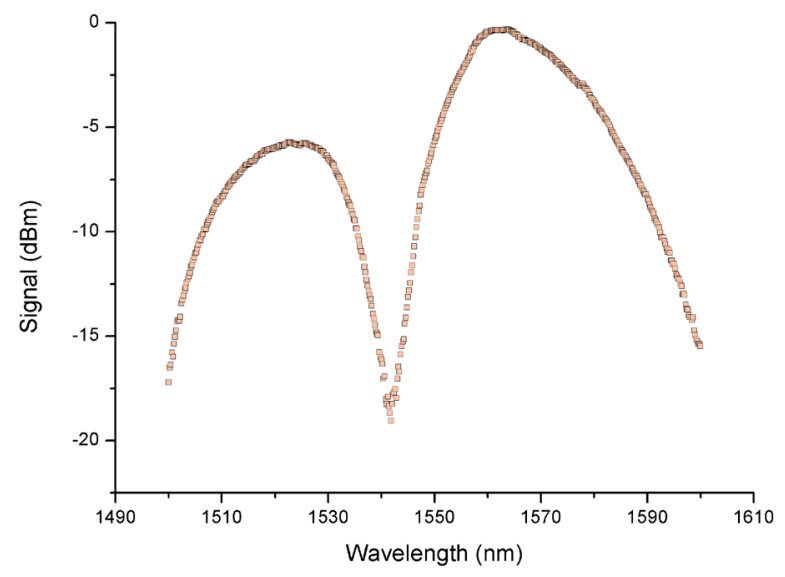

Fig. 8 Transmission spectrum of the SR-BG waveguide with $15 \mathrm{dBm}$ dip and minimum at wavelength $1540 \mathrm{~nm}$

\section{Conclusion}

In this paper we presented the results achieved using siloxane based technologies. Using the laser interference lithography in Mach-Zehnder configuration we prepared different 2D grating structures in periodical and quasiperiodical. By combining PDMS and PDMDPS embossing techniques we achieved 2D structures for the on-chip applications in optics and optoelectronics. We designed thin polymer membranes with patterned surface using $\mathrm{PhC}$ and $\mathrm{PQC}$ structures as an effective diffraction element for improving light extraction efficiency and modification of radiation pattern diagram of LEDs. The effect of structures was investigated by the near field measurement from orange emitting LED. Also, the thin PDMS membrane was used for the enhancement of angular detection properties of GaAs -based photodiodes. The combination of PDMDPS and PDMS was used as an alternative material for fabrication of planar photonic devices using waveguide with surface relief Bragg grating. We showed the transmission spectrum of this waveguide with proposed spectral dip. All these results favor the siloxane membranes and the used technologies for using in polymer photonics.

\section{Acknowledgement}

This work was supported by the Slovak National Grant Agency under the projects No. VEGA 1/0491/14 and 1/0278/15 and the Slovak Research and Development Agency under the project No. APVV 0395-12. 


\section{References}

[1] CAI, Z., QIU, W., SHAO, G., WANG, W.: A New Fabrication Method for All-PDMS Waveguides. Sensors and Actuators A., 204, 44-47, 2013.

[2] MARTINCEK, I., PUDIS, D., CHALUPOVA, M.: Technology for the Preparation of PDMS Optical Fibers and Some Fiber Structures. IEEE Photonisc Technology Letters, 26, 1446-1449, 2014.

[3] PAEK, J., KIM, J.: Microsphere-Assisted Fabrication of High Aspect-Ratio Elastomeric Micropillars and Waveguides. Nature Communications, 5, 3324, 2014.

[4] JIANG, S., et al: Study on Light Extraction from GaN-Based Green Light-Emitting Diodes Using Anodic Aluminum Oxide Pattern and Nanoimprint Lithography. Scientificreports, 6, 21573, 2016.

[5] CHANG, Y., LIOU, J., LIU, W.: Improved Light Extraction Efficiency of a High-Power GaN-Based Light-Emitting Diode with a Three-Dimensional-Photonic Crystal (3-D-PhC) Backside Reflector. IEEE Electron Device Letters, 34, 777-779, 2013.

[6] LAI, CH., HSIEH, CH., WU, CH.: Light-Spectrum Modification of Warm White-Light-Emitting Diodeswith 3D Colloidal Photonic Crystals to Approximate Candle Light. Optics Letters, 38, 3612-3615, 2013.

[7] PUDIS, D., et al: PDMS-Based Nanoimprint Lithography for Photonics. Communications-Scientific Letters of University of Zilina, 16(1), 15-19, 2014.

[8] Sylgard 184 Silicone Elastomer [online]. Date: 17/03/2017. Available at: http://www.dowcorning.com/DataFiles/090276fe80190b08. pdf.

[9] LS-6943 optical thermoset [online]. Date: 17/03/2017. Available at: https://nusil.com/services/downloadfile.ashx?productcode=LS6943\&originalname=LS-6943P.pdf.

[10] SKRINIAROVA, J., et al.: Periodic Structures Prepared by Two-Beam Interference Method. Microelectronics Journal, 38, 746-749, 2007.

[11] PUDIS, D., et al: Effect of 2D Photonic Structure Patterned in the LED Surface on Emission Properties. Applied Surface Science, 269, 161-165, 2013.

[12] PUDIS, D., et al: Siloxane Based Photonic Structures and Their Application in Optic and Optoelectronic Devices. Proc. of SPIE: Wave and Qquantum Aspects of Contemporary Optics, Slovakia, 10142, 2016.

[13] PUDIS, D., et al: GaAs-Based Photodetector with Applied PDMS Membrane with Photonic Crystal in the Surface. Conference on Advanced semiconductor devices and microsystems (ASDAM) 2016, Slovakia, 33-36, 2016. 